\title{
Demand Side Management using Profile Steering
}

\author{
Marco E. T. Gerards, Hermen A. Toersche, Gerwin Hoogsteen, \\ Thijs van der Klauw, Johann L. Hurink, Gerard J. M. Smit \\ Department of EEMCS \\ University of Twente \\ Enschede, the Netherlands
}

\begin{abstract}
Many Demand Side Management (DSM) approaches use energy prices as steering signals. This paper shows that such steering signals may result in power quality problems and high losses. As an alternative, this paper proposes to use desired (e.g., flat) power profiles as steering signals and presents an efficient scheduling algorithm that can follow desired power profiles. This paper investigates the complexity of price and profile steering, and presents an algorithm for profile steering.

The evaluation of this algorithm studies the results of a best possible uniform pricing and profile steering for a case of 121 houses, each with an electrical vehicle of which the power consumption can be controlled and shifted in time. In contrast to the other evaluated approaches, our profile steering algorithm results in a much flatter profile and keeps the voltage between $220 \mathrm{~V}$ and $235 \mathrm{~V}$ at each node. It reduces distribution losses by $57 \%$ compared to no control, and by $48 \%$ compared to uniform pricing.

Index Terms-Power quality, scheduling, smart grids.
\end{abstract}

\section{INTRODUCTION}

Electricity networks are dimensioned to accommodate expected peak loads. Existing networks were often planned decades ago and not designed for a high penetration of large loads, such as electrical vehicles (EVs) and heat pumps, and for large decentralized generation by, e.g., PV. As these devices increase the peak load of the consumption or generate peaks in production, measures such as peak shaving are an interesting alternative to costly grid reinforcements. Reducing peaks has additional advantages, such as: reduction of transport losses, power quality improvements and reducing the need for peak generators. Siano [1] surveys many reasons for peak shaving and goals of Demand Side Management (DSM) in general. Many of the current approaches only aim at peak shaving at the transformer, and do not consider power quality problems within the network. In contrast, our DSM approach prevents peaks in each hierarchical level of the electricity grid, and therefore resolves power quality problems at each level.

For controlling the power flows, many DSM approaches use price signals as steering signals. In Section II we argue that by using such signals, the peaks can get even higher than without control. Instead, we propose the use of desired power profiles as the steering signal (referred to as profile steering). A (desired)

This research was conducted within the e-balance project that has received funding from the European Union Seventh Framework Programme (FP7/2007 2013) under grant agreement $n^{\circ}[609132]$, the DREAM project supported by STW (\#11842), and the EASI project supported by STW (\#12700) and Alliander. power profile is a list of power values, e.g., $24 \times 4=96$ power values for a power profile of one day where each value represents a 15 minute interval. In our approach, we make a day-ahead planning for controllable appliances based on predictions of customer behavior/preferences, production and consumption behavior. This planning is subsequently followed until a new planning is initiated, because the old one is no longer achievable, e.g., when a prediction is updated or incorrect.

In this paper we present an algorithm that schedules a group of houses, or a group of appliances. Hereby we used tailored algorithms to create schedules for the individual appliances, e.g., we use the algorithm by van der Klauw et al. [2] to schedule EVs. The scheduling algorithm that we present has two phases: an initialization phase and an iterative phase. During the initial phase, each house is asked to steer its own profile toward a flat profile (or some other balancing of loads). After this phase, the achieved power profile at the neighborhood level is in general already relatively flat. In the iterative phase, the power profiles of all houses are collected, summed up and the deviation (euclidean distance) from a flat profile is calculated. Such deviations are used as steering signals for the houses in the next iteration.

Section II presents a background on both price and profile steering. The complexity of these steering methods is researched in Section III, wherein it is proven that price and profile steering are both NP-hard. As a consequence, for efficiency reasons, we require heuristic algorithms for profile steering. In Section IV we present such a heuristic algorithm. Section V compares optimal uniform pricing with our profile steering algorithm, and contains an evaluation of our algorithm. This evaluation shows that our algorithm keeps the voltages within the legal bounds and it reduces the losses significantly.

To summarize, the main contributions of this paper are:

- An NP-hardness proof of both price and profile based scheduling (Section III);

- An easy to implement iterative profile steering algorithm (Section IV);

- A comparison of optimal uniform pricing with profile based scheduling (Section V).

\section{BACKGROUND AND RELATED WORK}

Before introducing our results, we provide some background knowledge and introduce some notation. In Section II-A we 
discuss the problems which may occur when using price signals and subsequently in Section II-B we discuss our approach of using desired power profiles as steering signals.

\section{A. Price steering}

Within most DSM approaches, an aggregator can steer a group of houses towards a given objective using steering signals. These steering signals are often in the form of time varying prices for a unit of energy. This approach, referred to as dynamic pricing, is a well accepted and popular approach in the research literature and in practice (e.g., [3]-[6]). The response of a house to such a signal consists of shifting its consumption from where it is relatively expensive to where it is relatively cheap.

When all households receive the same price signal (referred to as uniform pricing), all houses tend to shift their loads to the periods where the energy is cheapest. McKenna and Keana [3] show that, under several uniform pricing schemes that are used to motivate customers to shift their load, the peaks are hardly reduced and only shifted in time. Moreover, in this paper we show that by using control algorithms, these problems may even become larger. The problem increases even further in future scenarios where customers have a lot of PV, EVs and heat pumps installed. Hence, uniform pricing is not a proper approach for peak shaving.

One way to circumvent this specific problem and to do proper peak shaving on neighborhood level is differentiated dynamic pricing (see e.g. [4]), which uses different price signals for individual houses. This causes customers to create peaks at different times, alleviating the aforementioned problem of creating new peaks at the neighborhood level. However, as a result, each house tends to a separate (local) peak. Differentiated dynamic pricing will balance these peaks such that they cancel at the transformer level. These peaks usually imply that at a house level, the production and consumption are not balanced, nor spread out over the day. Furthermore, the loads between phases in the grid are likely not properly balanced. Since this may result in voltage problems and overloading of cables, high investments in cables to houses may still be required. In other words, differentiated dynamic pricing may not solve the problem appropriately either.

The underlying problem with dynamic pricing is that it motivates extreme behavior: the households get a request to increase (low price) or decrease (high price) its load at specific time intervals, hence the steering signals are vague ("more" and "less") while the goal (the actual desired load) is clear and available. The resulting power quality problems can be addressed by fine-tuning differentiated dynamic pricing algorithms [7], and using detailed information on the grid topology. Note, that the goal of our paper is to avoid these problems altogether, by reconsidering the steering signals that are used for DSM.

Another way to improve dynamic pricing is to make the price dependent on the amount bought (i.e., nonlinear pricing), such that there is no incentive to move all consumption to a certain time window. Such an approach is used by the PowerMatcher (see, e.g., [8], [9]), which uses an energy auction between the different agents. Just as in our approach, an incentive is given for balancing, and not for increasing or decreasing the consumption arbitrarily. However, whereas our planning-based approach takes the future into account and may allow a small peak at the current time such that flexibility is preserved to reduce a big peak load at a later time, the PowerMatcher only considers the current balance within the network without explicitly considering the future. Furthermore, our approach shaves peaks at several hierarchical levels of the grid (e.g., house, LV grid, MV grid, etc.). We refer to the paper by Molderink et al. [10] for an in-depth discussion about planning-based approaches versus online approaches such as the PowerMatcher.

\section{B. Profile steering}

Demand side management is used to shape the production and/or consumption of electricity within a house or neighborhood in some way. This is done by means of controllable appliances such as time-shiftable washing machines, electrical vehicles and batteries. For the following explanations, we take the washing machine as an example.

Most appliances cannot be controlled freely, for example, controllable washing machines that are available on the market have a fixed profile that can only be shifted in time. To what extent such appliances can be time-shifted depends on the configuration by the user, which states when the appliance is available (called "arrival time") and how long it may be deferred. In general, restrictions on the flexibility of an appliance are called flexibility constraints.

For the washing machine, the DSM software decides when it is started, respecting the flexibility constraints. After such a planning decision is made (i.e., a start time is chosen), the resulting power profile for the washing machine can be calculated.

More precisely, the planning decision for the washing machine is given by a single variable $s$, which is the start time of the appliance. The start time should be at least the arrival time $a$, and at most $a+D$ ( $D$ is the maximal deferral time), hence: $a \leq s \leq a+D$. We denote the power consumption of the washing machine in its $n$-th interval (e.g., for intervals of 15 minutes) relative to its start time by $P_{n}^{\mathrm{WM}}$, where $P_{n}^{\mathrm{WM}}=0$ when the washing machine is not active in interval $n$. Now the power profile for the washing machine is given by

$$
x_{n}^{\mathrm{WM}}=P_{s-n}^{\mathrm{WM}} .
$$

Summing the power profiles for all appliances within a house yields the household power profile $\vec{x}$ or obtained profile. A planning algorithm within a DSM approach now chooses the states (e.g., the start time $s_{m}$ for the washing machine) such that the obtained profile $\vec{x}=\left[x_{1}, \ldots, x_{N}\right]^{T}$ ( $N$ is the number of intervals) minimizes the costs for a given steering signal.

Whereas price steering indicates in which intervals the consumption / production should be increased or decreased as much as possible, we argued in the previous section that it is desirable to use a steering signal that precisely indicates what the goal is. Such steering signals can be (deviations from) a desired power profile, which is used by the approach presented in this paper. We refer to this approach as profile steering. 
More precisely, with profile steering we aim to minimize the distance between some desired profile $\vec{p}=\left[p_{1}, \ldots, p_{N}\right]^{T}$ (e.g., a flat power profile) and the obtained profile $\vec{x}$ that results from the planning algorithm, while respecting all the appliance constraints. This distance between $\vec{p}$ and $\vec{x}$ can be obtained using any arbitrary vector norm, denoted by $\|\vec{x}-\vec{p}\|_{*}$. A particular example of a distance measure is the euclidean distance, commonly denoted by $\left.\|\vec{x}-\vec{p}\|\right|_{2}$. We use the euclidean distance in our evaluation, because in practice it has a good performance.

The remainder of this paper discusses the inherent complexity of the profile steering problem, and provides and evaluates an algorithm for profile steering.

\section{COMPLEXITY}

When the steering signal for a given house is known, a planning algorithm can be used to find the optimal planning for all appliances within the house. This section studies the complexity of this planning for a specific sub-problem, namely for multiple time-shiftable appliances. This problem is formally stated as follows.

Problem 1 (Optimal planning of time-shiftable appliances). Let there be $N$ time intervals, $M$ time-shiftable appliances, and each appliance $m \in\{1, \ldots, M\}$ is active for $T_{m}$ intervals and has a power consumption $P_{m, n}$ within the $n$-th interval relative to its (to be determined) starting interval $s_{m}$. For given starting intervals $s_{1}, \ldots, s_{M}$, the total power for interval $n$ is given by $x_{n}=\sum_{m=1}^{M} P_{m, n-s_{m}}$. The problem is to determine the starting intervals $s_{1}, \ldots, s_{M}$ such that they minimize the total costs given by

$$
\sum_{n=1}^{N} f_{n}\left(x_{n}\right),
$$

for some (to be defined) cost function $f_{n}$ under the restriction that

$$
x_{n} \leq P^{M A X}, \text { for all } n .
$$

Note, that the steering signals are used to specify the concrete cost functions. Furthermore, note that it is already NP-complete to determine whether a feasible solution to this problem exists is NP-complete (i.e., for every possible steering signal), since it is already hard to keep the power within each interval below $P^{\mathrm{MAX}}$. This is stated formally by the following theorem.

Theorem 1. Determining if a feasible solution to "Optimal planning of time-shiftable appliances" exists is already NPcomplete for instances with two time intervals $(N=2)$ and appliances which only run within one time interval $\left(T_{m}=1\right)$.

Proof. The problem is obviously in the class NP.

To prove NP-completeness, we derive a reduction from the NP-complete partitioning problem ${ }^{1}$ as follows. For a given multiset $S=\left\{x_{1}, \ldots, x_{K}\right\}$ of the partitioning problem we construct the following instance of Problem 1: $M=K, N=2$,

\footnotetext{
${ }^{1}$ The partitioning problem considers a given multiset $S$ of positive integers that must be partitioned into two subsets $S_{1}$ and $S_{2}$, such that the sums of the elements in both sets are equal.
}

$T_{m}=1, P_{m, 1}=x_{m}$ for all $m \in\{1, \ldots, M\}$, and $P^{\mathrm{MAX}}=$ $\frac{1}{2} \sum_{m=1}^{M} x_{m}$. A feasible solution $\left\{s_{1}, \ldots, s_{M}\right\}$ of Problem 1 can be translated to a feasible solution of the partitioning problem using:

$$
\begin{aligned}
& S_{1}=\left\{x_{m} \in S \mid s_{m}=1\right\}, \\
& S_{2}=\left\{x_{m} \in S \mid s_{m}=2\right\},
\end{aligned}
$$

and vice versa. Since this is a polynomial time reduction of the partitioning problem to Problem 1, and since Problem 1 is in NP, it directly follows that the theorem holds.

In Theorem 1 we used $P^{\mathrm{MAX}}$ to prove NP-completeness independent of the cost functions $f_{n}$. However, it can also be proven that Problem 1 is NP-hard for a (large and relevant) set of cost functions $f_{n}$ without a bound $P^{\mathrm{MAX}}$.

These complexity results imply that it is computationally hard to calculate the optimal solution in many situations, especially on an embedded computer with a relatively small memory and a relatively slow processor. Therefore, we aim at finding a heuristic. In the next section we give such a heuristic algorithm for profile steering.

\section{Algorithm}

This section treats a profile-steering based approach to simple case of a single appliance first (Section IV-A), followed by a discussion on the multiple appliance case (Section IV-B).

\section{A. Single appliance}

Before covering the case of multiple appliances $(M>1)$, we first cover the case of a single appliance $(M=1)$. A corresponding planning algorithm determines all future decisions for this appliance (i.e., its future states), which eventually results in a power profile $\vec{x}$.

An example of an appliance is a time-shiftable appliance such as a washing machine for which the optimal starting time $s$ must be determined. This can be easily done, namely by considering all possible (discrete) starting times that are allowed and choosing the one which gives a power profile nearest to the desired profile. Note, that it is important to take the restrictions of the appliance into account, such as the window wherein the appliance should run.

For several appliances, tailored algorithms have been developed that minimize the distance toward a desired profile. An example of such an algorithm is the EV planning algorithm by van der Klauw et al. [2], which takes charging power restrictions and a deadline into account.

\section{B. Multiple appliances}

Profile steering for multiple appliances is in general NP-hard (see Section III), hence a heuristic algorithm must be used. This section presents such a heuristic algorithm, which is capable of planning a set of houses, or a set of appliances within a house. To ease the discussion, we explain the algorithm from the perspective of a house, with multiple appliances that are planned as discussed in Section IV-A. Note, that this algorithm is not restricted to a set of appliances within a house. It works on every level in the hierarchy, for example in a neighborhood 
to steer multiple houses (i.e., replace "house" by "neighborhood" and "appliance" by "house").

Initially, the algorithm asks each appliance to produce a smooth profile while respecting its constraints, i.e., to obtain some profile that is as close as possible to the zero profile. Next, the sum of the power profiles of all appliances $\vec{x}=$ $\sum_{m=1}^{M} \overrightarrow{x_{m}}$ is then calculated, and subsequently the difference $\vec{d}$ between this vector $\vec{x}$ and the desired planning on a household level $\vec{p}$ is determined. Now, each appliance $m$ reports how much it can reduce the distance $\left(\|\vec{d}\|_{2}\right)$ between the actual (i.e., obtained) and desired planning of a house by replacing its current planning with a new candidate planning $\overrightarrow{\hat{x}}_{m}$. The appliance with the largest improvement is selected, and this appliance updates its planning to its candidate planning $\overrightarrow{\hat{x}}_{m}$. This process is repeated iteratively. The details of this algorithm are presented in Algorithm 1.

This planning algorithm can be applied in each level of the grid hierarchy, starting with the top level. The advantage of this approach is that first a locally flat planning is made. For example, the power consumption of each individual house is flattened as much as possible. Only when this is not sufficient, and peaks occur at the neighborhood level, the neighborhood planner asks the houses in this neighborhood to compensate for this problem.

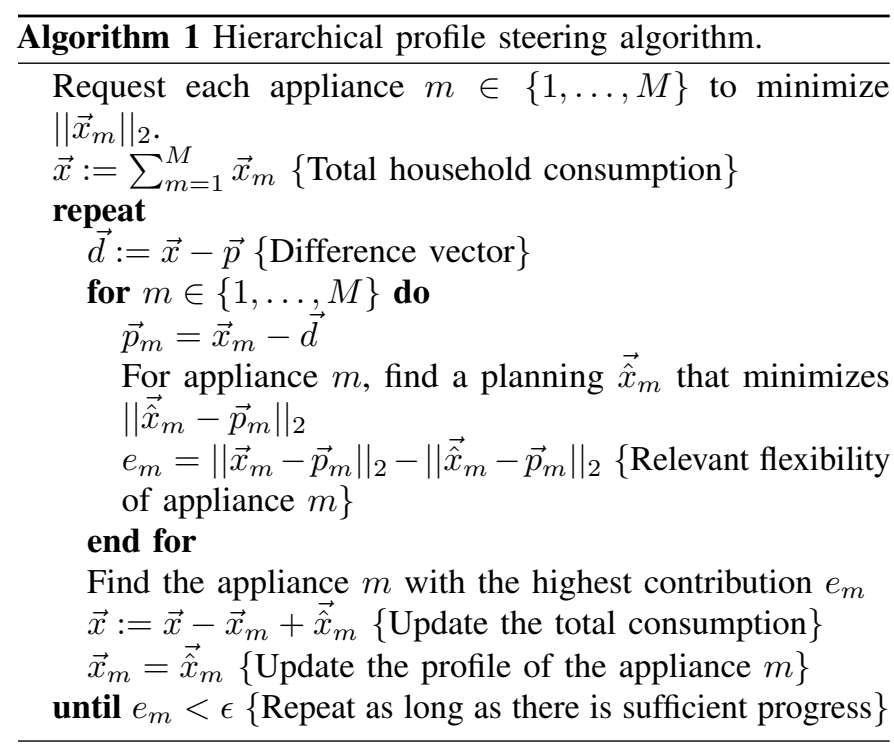

A few design decisions require special attention. We have chosen to select only a single appliance in each iteration. Using two (or more) appliances has a risk of a worse performance, because together they may overcompensate, which results in a worse profile than when using a single appliance.

Another major design decision is the use of the 2-norm. It is possible to use any other norm in this algorithm, but for minimizing the 2-norm under flexibility constraints there are computationally efficient algorithms (e.g., quadratic programming). Furthermore, as the losses have a close correspondence to the squared power, the 2-norm has a good performance in terms of power quality and losses as we demonstrate in the next section. Note, that the 2-norm directly minimizes the absolute deviation from some desired power profile as is commonly used to evaluate demand side management approaches (see, e.g., [10]).

\section{EVALUATION}

This section evaluates the performance of the algorithm from Section IV. First, we compare our profile steering algorithm with a best possible uniform pricing. For this, we derived a specific case (described in Section V-A) for which we are able to determine the uniform prices, which lead to the best possible reaction of the appliance. We call these the optimal uniform prices. Second, we compare our profile steering algorithm to the state-of-the-art algorithm using a more extensive scenario (Section V-B).

\section{A. Optimal uniform pricing}

In this section, we compare optimal uniform pricing (all houses receive the same price signal) with profile steering. As reference, we consider the case where the aggregator sends the globally optimal price signal to each house. To be able to determine the optimal prices, we have chosen to only consider EVs, which all must charge 12 kWh between 18:00 and 07:00 with a charging power between $0 \mathrm{~kW}$ and $3.8 \mathrm{~kW}$.

To study the effects of price and profile based DSM approaches on a highly loaded electricity network, we determine the optimal prices and compare the resulting profiles with our profile steering algorithm. We used a detailed (three phases) network model of a dutch LV grid with 121 houses (with real measurements), and we added to each house a steerable EV. For this we calculated the load flows to obtain the power at the transformer, voltages within the network and transport losses.

The first case we study is the extreme case where all EVs are charged at full power as soon as they arrive at home (at 18:00). The dotted blue line in Fig. 1 shows the total power (including losses) at the transformer. Since some cables are overloaded (not explicitly shown in the figures), and because the voltages are almost out of their legal bounds (i.e., NEN-EN 50160:2010 [11], $207 \mathrm{~V}-253 \mathrm{~V}$ ), a different planning is desired. Besides these power quality problems, also the transport losses of $89.6 \mathrm{kWh}$ during this single day ( $3.2 \%$ of the total $2759.3 \mathrm{kWh})$ are large.

With optimal uniform pricing, the peak is only shifted in time and is hardly reduced (the dashed red line in Fig. 1). Although the voltage is within the permitted bounds, it is still close to the permitted lower bound (Fig. 2b). Due to the slightly improved balance, the losses decreased to $73.6 \mathrm{kWh}(2.7 \%$ of the total).

As profile steering gives an incentive to move the load away from the peak, the peak is significantly reduced by our profile steering algorithm (the solid black line in Fig.1). Also, the voltage is well within the permitted bounds (Fig. 2c). As the loads are well balanced and flattened, the losses have decreased significantly to $38.1 \mathrm{kWh}(1.4 \%$ of the total).

\section{B. Comparison with state-of-the-art research}

Whereas the previous section compares profile steering with optimal uniform pricing, this section compares profile steering 


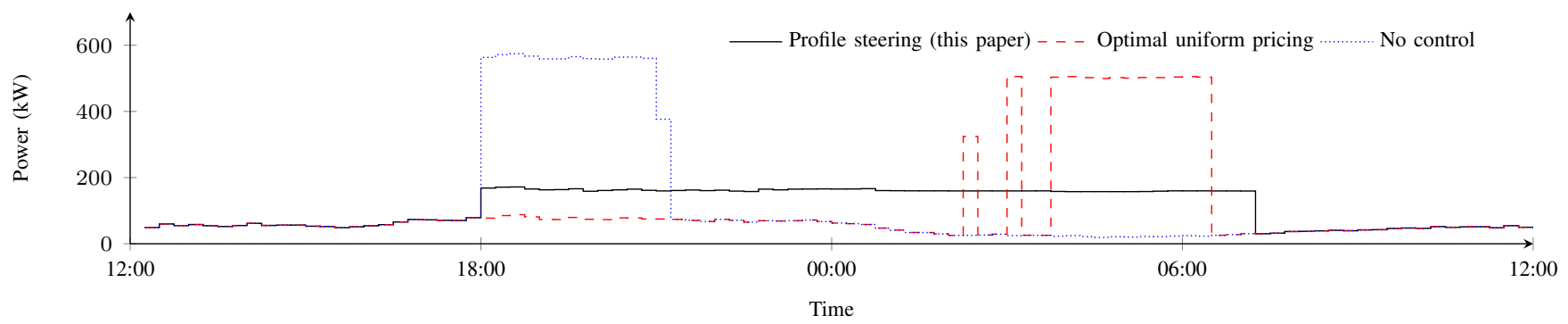

Fig. 1. Power at transformer (incl. losses) in the optimal uniform pricing case study

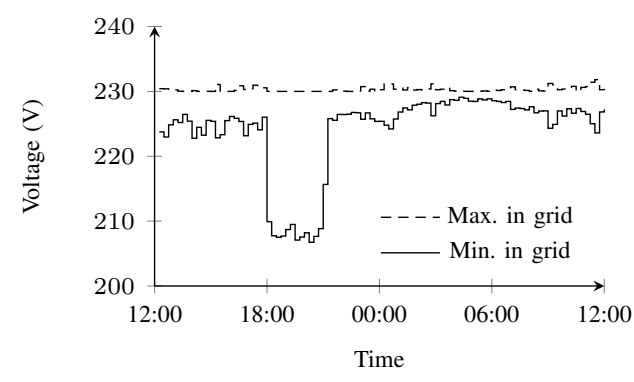

(a) EV charging at arrival

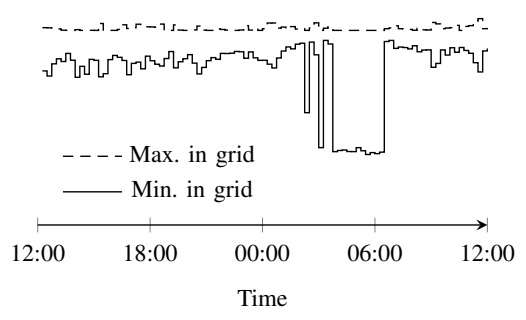

(b) EV charging at cheapest intervals

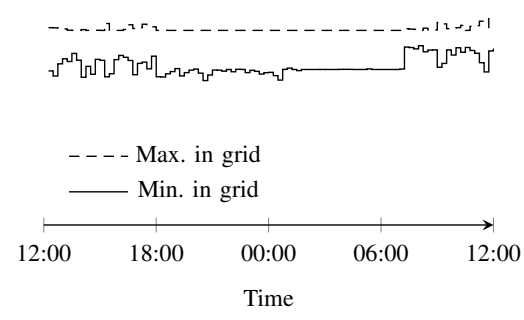

(c) Profile steering (this paper)

Fig. 2. Lowest and highest observed voltage for optimal uniform pricing case study

with the state-of-the-art research [7], which uses differentiated dynamic pricing. Both approaches are compared using a case study which has 121 houses, each with an EV (as in the previous section), a $3 \mathrm{kWh}$ battery $(3.7 \mathrm{~kW})$, solar panels $\left(12 \times 1.65 \mathrm{~m}^{2}\right.$, $18 \%$ efficiency, facing south) for which we use dutch weather data, and a time-shiftable washing machine (to be scheduled between 08:00 and 17:00). For this case, we use Algorithm 1 at two levels of the grid hierarchy, namely at the LV grid level and at the house level.

Without any control, all appliances are scheduled to start when they become available. This results in a peak at the transformer (the dotted blue line in Fig. 3), overloaded cables (see Table I), and the minimum observed voltage in the network drops below $210 \mathrm{~V}$ for several time intervals, as is shown in Fig. 4a.

When we use the DSM algorithms from [7], the situation improves significantly. The peak is flattened (dashed red line in Fig. 3), and the voltage remains within the permitted limits (Fig. 4b).

When we compare this to the profile steering approach that was introduced in this paper, we can see that the peak is also flattened (the solid black line in Fig. 3), and the voltage remains within the permitted limits (Fig. 4c). In fact, profile steering is better capable of decreasing the peaks, and keeping the voltages within the permitted interval. Note, that one important reason for DSM is to keep the cable load low such that the expected life time of the cable increases. Whereas the stateof-the-art approach keeps the load on all cables below 53\% of their capacity, our algorithm keeps the load on all cables below $32.1 \%$ of their capacity. For a numerical comparison between the state of the art and our profile steering algorithm,
TABLE I

COMPARISON BETWEEN DSM APPROACHES AND NO CONTROL

\begin{tabular}{lrrr}
\hline & No control & State of the art & Profile steering \\
\hline Total losses (kWh) & 26.6 & 3.0 & 1.5 \\
Lowest voltage (V) & 201.8 & 219.6 & 223.2 \\
Highest voltage (V) & 236.9 & 241.5 & 235.7 \\
Max. peak (kW) & 563.0 & 174.8 & 131.2 \\
Cable load (\%) & 143.2 & 53.0 & 32.1
\end{tabular}

see Table I.

Another advantage of our algorithm is that it yields a more stable situation, as can be seen by comparing Fig. $4 \mathrm{~b}$ and Fig. 4c. The reason for this is that the price signals used in [7] result in aggressive steering, whereas our algorithm keeps the system state stable for a relatively long time. In our approach we used the EV planning algorithm from [2], which assumes that all charging powers within the specified bounds are available. When the algorithm would take a more realistic range into account, our steering will also become slightly more aggressive. Another important difference between both algorithms, is that [7] requires detailed information of the network, while our algorithm does not require this information and still achieves better results because it shaves the peaks at each level in the grid. We only used the network topology after completion of the algorithm to calculate the voltages and losses that we presented in this evaluation, while the state-of-the-art research uses the topology in the optimization algorithm.

\section{Conclusions}

Peak shaving at all levels in the grid has a positive effect on the power quality and reduces the losses. We have argued that 


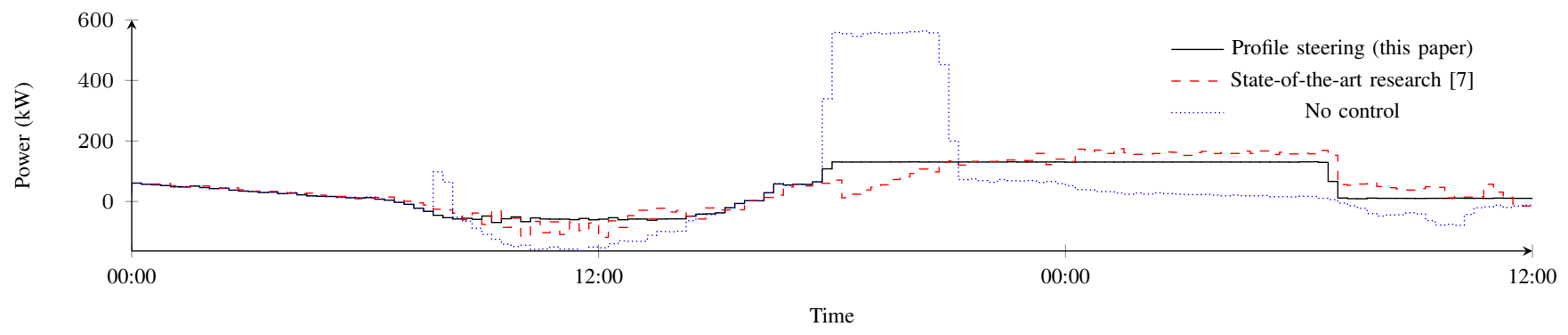

Fig. 3. Power at transformer (incl. losses) in the differentiated pricing case study

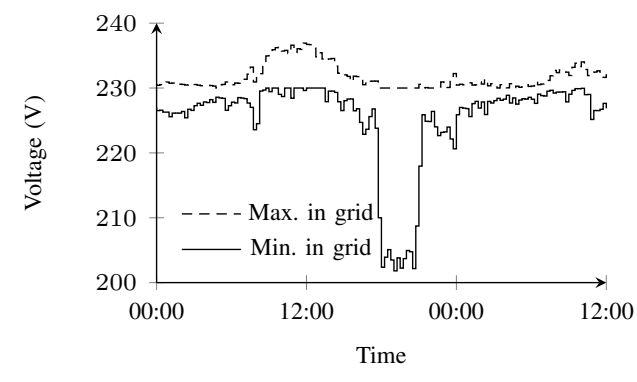

(a) No control

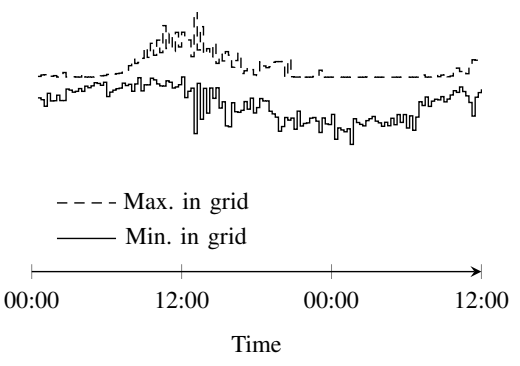

(b) State-of-the-art research

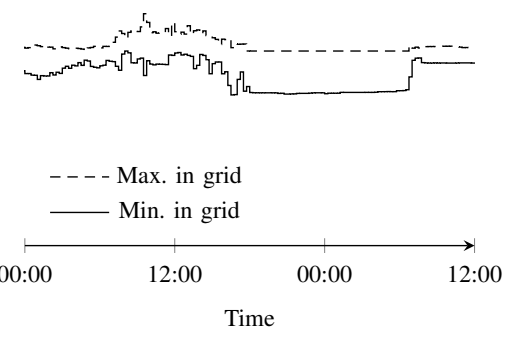

(c) Profile steering (this paper)

Fig. 4. Lowest and highest observed voltages in the differentiated pricing case study

the standard approach, which sends the same price signal to all houses, does not have the intended effect, since it does not peak shave at the house level. Instead, we proposed using (deviations from) desired profiles as steering signals and presented an efficient iterative algorithm for this problem. This algorithm first balances the load within a house, and then iteratively balances the loads in the electricity grid. Due to the initial local balancing, our algorithm has a fast convergence.

Our algorithm lowers the peaks significantly (locally and at the transformer), balances loads properly within the network, keeps voltages well within the legal bounds, and reduces the losses. The losses are reduced by $57 \%$ with respect to the case where EVs are charged at full power when they arrive, and by $48 \%$ with respect to the case of optimal uniform pricing. This indicates that profile steering can significantly improve the power quality and reduce the losses.

Compared to the state-of-the-art algorithm, our algorithm results in better peak shaving and a better power quality. Contrary to the state of the art, our algorithm does not require knowledge of the grid topology. Instead, it aims at peak shaving at each hierarchical level of the grid, which automatically results in a good power quality throughout the grid.

In future work, we apply this algorithm in a large case study and propose a market mechanism for profile based steering.

\section{REFERENCES}

[1] P. Siano, "Demand response and smart grids-a survey," Renewable and Sustainable Energy Reviews, vol. 30, pp. 461-478, 2014.
[2] T. van der Klauw, M. E. T. Gerards, G. J. M. Smit, and J. L. Hurink, "Optimal scheduling of electrical vehicle charging under two types of steering signals," in IEEE PES Innovative Smart Grid Technologies Conference Europe (ISGT-Europe), Istanbul, Turkey. USA: IEEE Power \& Energy Society, October 2014, pp. $1-6$.

[3] K. McKenna and A. Keane, "Discrete elastic residential load response under variable pricing schemes," in IEEE PES Innovative Smart Grid Technologies Conference Europe (ISGT-Europe), Istanbul, Turkey. USA: IEEE Power \& Energy Society, Oct 2014, pp. 1-6.

[4] A. Molderink, V. Bakker, M. G. C. Bosman, J. L. Hurink, and G. J. M. Smit, "Management and control of domestic smart grid technology," IEEE transactions on Smart Grid, vol. 1, no. 2, pp. 109-119, September 2010.

[5] A. Faruqui, D. Harris, and R. Hledik, "Unlocking the $€ 53$ billion savings from smart meters in the EU: How increasing the adoption of dynamic tariffs could make or break the EUs smart grid investment," Energy Policy, vol. 38, no. 10, pp. 6222-6231, 2010. [Online]. Available: http://www.sciencedirect.com/science/article/pii/S0301421510004738

[6] S. Bu, F. Yu, and P. Liu, "Dynamic pricing for demand-side management in the smart grid," in Online Conference on Green Communications (GreenCom), 2011 IEEE, Sept 2011, pp. 47-51.

[7] G. Hoogsteen, A. Molderink, J. L. Hurink, and G. J. M. Smit, "Managing energy in time and space in smart grids using triana," in Proceedings of the IEEE PES Innovative Smart Grid Technologies Conference Europe, ISGT-Europe 2014, Istanbul, Turkey. USA: IEEE Power \& Energy Society, October 2014, pp. 1-6.

[8] K. Kok, "Dynamic pricing as control mechanism," in Power and Energy Society General Meeting, 2011 IEEE, July 2011, pp. 1-8.

[9] _ _, "The powermatcher: Smart coordination for the smart electricity grid," Ph.D. dissertation, Vrije Universiteit Amsterdam and TNO, 2013.

[10] A. Molderink, V. Bakker, J. L. Hurink, and G. J. M. Smit, "On indirect controlled cost function based DSM strategies," in Proceedings of IEEE PowerTech (POWERTECH), Grenoble. USA: IEEE Power \& Energy Society, June 2013, pp. 1-6.

[11] NEN-EN 50160:2010, "Voltage characteristics of electricity supplied by public distribution network," 2010. 